\title{
ESR Observation of Methacrylate Propagating Radicals in Stationary State
}

\author{
Mikiharu KAMACHI, Masahiro KoHNO,* \\ Yoko KUWAE, and Shun-ichi NOZAKURA \\ Department of Macromolecular Science, Faculty of Science, \\ Osaka University, Toyonaka, Osaka 560, Japan \\ *Japan Electron Optics Laboratory Company, \\ Akishima, Tokyo 196, Japan
}

(Received March 2, 1982)

\begin{abstract}
KEY WORDS ESR / Propagating Radical / Stationary State / Methyl Methacrylate / iso-Butyl Methacrylate / Benzyl Methacrylate / Triphenylmethyl Methacrylate / Propagation Rate Constant / Termination Rate Constant /
\end{abstract}

Considerable data on the propagation and termination rate constants $\left(k_{\mathrm{p}}\right.$ and $\left.k_{\mathrm{t}}\right)$ in the radical polymerization of vinyl compounds have been reported so far. ${ }^{1}$ These data were obtained primarily by the rotating sector method, non-stationary measurements (pre-effect or after-effect), the viscosity method, and the spatial interference method. The estimation of $k_{\mathrm{p}}$ and $k_{\mathrm{t}}$ from these indirect measurements is inevitably accompanied by large error. This explains the considerable scattering of data obtained by various authors. ${ }^{1}$ Moreover, the data was obtained on the assumption that the rate of polymerization is described by the normal kinetic equation of [Monomer]/[Initoator]. ${ }^{0.5}$ This prerequisite restricts the scope of indirect measurements to a limited number of vinyl monomers.

ESR spectroscopy is known to be possibly the best method for determining rate constants, since in principle stationary free radical concentration can be measured directly. Unfortunately, the stationary concentration of propagating radicals was too low to be detected by a commercial ESR spectrometer. Thus, ESR studies on propagating radicals were performed under special conditions such as the frozen state ${ }^{2,3}$ and the crystalline state, ${ }^{4,5}$ or by use of a special technique such as the flow technique. ${ }^{6-8}$ Since these conditions for measurement are quite different from those ordinarily used in radical polymerization, they are not applicable to the quanti- tative estimation of kinetic rate constants in radical polymerization.

In 1972, Bresler et al..$^{9,10}$ detected propagating radicals in the bulk polymerization system of vinyl compounds using a sensitive ESR spectrometer equipped with "a balance resonator," and estimated rate constants directly.

We have observed more highly resolved ESR spectra than Bresler's for the propagating radicals of methacrylates in solution using an ESR spectrometer equipped with a specially designed $\mathrm{TM}_{110}$ cavity. In the following, we report the ESR observation of the propagating radicals of methyl methacrylate (MMA) and its homologs in benzene at room temperature.

The purification of $\mathrm{MMA},{ }^{2}$ triphenylmethyl methacrylate (TPMA) ${ }^{12}$ and benzene ${ }^{11}$ has already been described. Benzyl methacrylate (BzMA), and isobutyl methacrylate ( $i \mathrm{BMA}$ ) were purified by the same method as MMA. ${ }^{2}$ Benzoyl peroxide was repeatedly recrystallized from ethanol and dried in vacuo.

After the initiator $(0.010 \mathrm{~g})$ was dissolved in $1 \mathrm{ml}$ of a mixture of methacrylate (MMA, $i \mathrm{BMA}$, or BzMA) and benzene (volume ratio=1:1), the solution for ESR spectrometry was placed under nitrogen in a flat cell $(3.5 \mathrm{~mm} \times 3.0 \mathrm{~mm}, 1.0 \mathrm{~mm}$ thickness). In the case of TPMA, the initiator $(0.010 \mathrm{~g})$ and TPMA $(0.316 \mathrm{~g})$ were dissolved in $1 \mathrm{ml}$ 
of benzene. The polymerization was carried out in an ESR spectrometer by irradiation through a slotted opening of the cavity with a 500-W high pressure mercury lamp (USHIO 500).

The extent of polymerization was determined by a precipitation method in which the formed polymer was precipitated with methanol, reprecipitated from benzene and methanol, freeze-dried from the benzene solution, and weighed. The extent of polymerization after 15 minutes was found to be

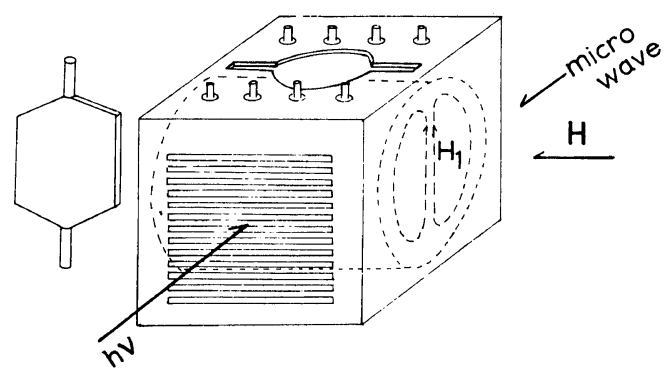

Figure 1. $\mathrm{TM}_{110}$ mode cavity improved for photoreaction.

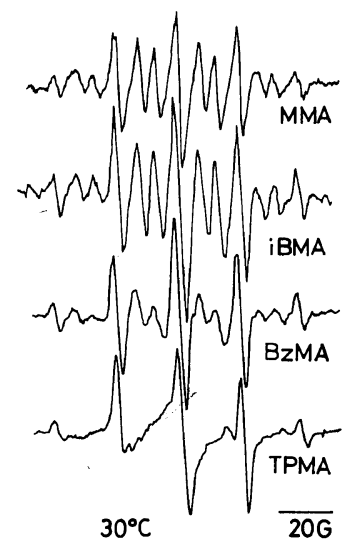

Figure 2. ESR spectra of propagating radicals of methylacrylates at $30^{\circ} \mathrm{C}$.

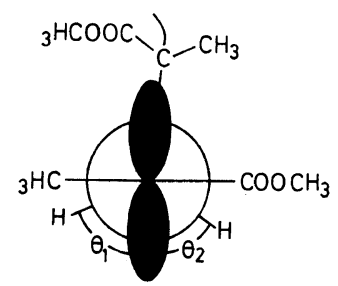

Figure 3. Conformation of the poly(MMA) radical at $30^{\circ} \mathrm{C} . \theta_{1}=65^{\circ}$ and $\theta_{2}=55^{\circ}$.
$4.1 \%$ and $1.8 \%$ for MMA and TPMA, respectively. The $\mathrm{TM}_{110}$ mode cavity improved for photopolymerization is shown in Figure 1. The use of $\mathrm{TM}_{110}$ mode made it possible for the light irradiation to be perpendicular to the flat cell surface through a slotted opening in the front side of the cavity. The sensitivity of the cavity for detection of free radicals was more than 10 times higher than that of a conventional $\mathrm{TE}_{011}$ mode cavity making it possible to detect radical concentration in the stationary state.

The ESR spectra of the propagating radicals of MMA, $i$ BMA, BzMA, and TPMA are shown in Figure 2. No ESR spectrum was observed without the initiator. An ESR spectrum of the initiating radical was found in the absence of methacrylate, but an ESR spectrum of the propagating radical could be detected only in the presence of the monomer. The $13(5+8)$-line spectra were observed for MMA, $i \mathrm{BMA}$, and BzMA, and the inner 8 lines almost disappeared in the ESR spectrum of the poly(TPMA) radical. The intensity distribution of the 13-line spectrum of the poly(MMA) radical is consistent with the theoretical distribution $(1: 1: 1$ : $4: 3: 3: 6: 3: 3: 4: 1: 1: 1)$ for the conformation in which dihedral angles of $\beta$-protons with the $\pi$ orbital of the unpaired electron are $\theta_{1}=55^{\circ}$ and $\theta_{2}=65^{\circ}$ (Figure 3). The inner 8 lines become weaker with an increase in the bulkiness of the ester group. This phenomenon is consistent with exchange broadening proposed to explain the change in the ESR spectrum of the poly(TPMA) radical in a temperature range from $0^{\circ} \mathrm{C}$ and $30^{\circ} \mathrm{C}^{12}$

The free radical concentration was determined by a graphical integration of the spectra and by calibration of the spectrometer using $1.00 \times 10^{-4} \mathrm{M}$ of 4-hydroxy-2,2,6,6-tetramethyl-piperidin-1-oxyl (Tempol) in benzene. From the free radical concentration, the value of $k_{\mathrm{p}}$ for MMA and TPMA was determined using the following equation for the steady state polymerization:

$$
R_{\mathrm{p}}=k_{\mathrm{p}}[\mathrm{M} \cdot][\mathrm{M}]
$$

where $R_{\mathrm{p}}$ is the polymerization rate, and [M] and [M.] are the concentrations of monomer and radical, respectively. The results are shown in Table I along with data obtained by the conventional method. ${ }^{11}$ The $k_{\mathrm{p}}$ for TPMA is smaller than that for MMA, which is possibly due to the steric effect of the bulky triphenylmethyl group on the propa- 
ESR Observation of Propagating Radical

Table I. Kinetic data for MMA and TPMA

\begin{tabular}{|c|c|c|c|c|c|c|}
\hline Monomer & Radical $\times 10^{7}$ & $R_{\mathrm{p}} \times 10^{5}$ & $k_{\mathrm{p}}$ & $k_{\mathrm{t}} \times 10^{-5}$ & $k_{\mathrm{p}}$ & $k_{\mathrm{t}} \times 10^{-5}$ \\
\hline $\mathbf{M}_{0}$ & $\mathbf{M}$ & $\mathrm{Ms}^{-1}$ & $M^{-1} s^{-1}$ & $M^{-1} s^{-1}$ & $\mathbf{M}^{-1} \mathrm{~s}^{-1}$ & $\mathrm{M}^{-1} \mathrm{~s}^{-1}$ \\
\hline MMA & 2.7 & 21.5 & 187 & - & $450^{\mathrm{a}}$ & $420^{\mathrm{a}}$ \\
\hline TPMA & 10.5 & 2.73 & 26 & 3.01 & - & - \\
\hline
\end{tabular}

a Data from literature 11.

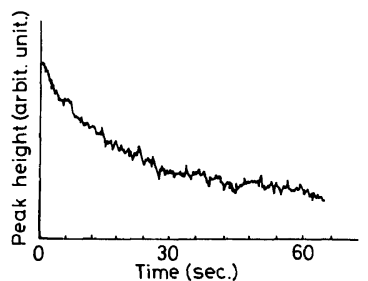

Figure 4. Decay curve of the poly(TPMA) radical after turning off light.

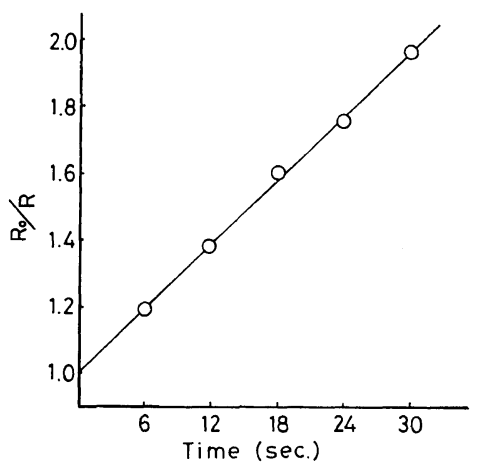

Figure 5. Second order plot of peak height after turning off.

gation rate. The value of $k_{\mathrm{p}}$ for MMA estimated in these data was about one third of the value obtained by the rotating sector method. ${ }^{11}$ The smaller value of $k_{\mathrm{p}}$ estimated by this work may possibly be ascribed to an underestimation of the polymerization rate, since estimation of this rate by the precipitation method is likely to overlook the presence of oligomers.

Radical decay after turning off the light was followed by a change in peak height of the central line of the ESR spectrum with time (Figure 4). The radical decay of TPMA was detectable in the fastest scan rate of the recorder $\left(360 \mathrm{~mm} \mathrm{~min}^{-1}\right)$. The $k_{\mathrm{t}}$ value for TPMA and the lifetime of the propagating radical were estimated to be $3.01 \times 10^{5} \mathrm{M}^{-1} \mathrm{~s}^{-1}$ and $15.8 \mathrm{~s}$, respectively, from the second order plot of the peak height (Figure 5). The decay of the poly(MMA) radical was too fast to be detected in the scan of the recorder, indicating that the $k_{\mathrm{t}}$ value is much larger than that for TPMA. Since the $k_{\mathrm{t}}$ value for MMA has been estimated to be $4.20 \times 10^{7}$ $\mathrm{M}^{-1} \mathrm{~s}^{-1}$ by the rotating sector method, ${ }^{11}$ the experimental results for MMA seem reasonable. The smaller $k_{t}$ value for TPMA, compared with that for MMA, may reasonably be ascribed to the interference of the termination reaction due to the bulkiness of the triphenylmethyl group of TPMA.

Additional research on other monomers is now being carried out.

Acknowledgements. We should like to thank professors Heimei Yuki and Koichi Hatada for providing the TPMA. This research was partly supported by Grant-in-Aid for Scientific Research from the Ministry of Education, Science and Culture of Japan.

\section{REFERENCES}

1. R. Korus and K. F. O'Driscoll, "Polymer Handbook," 2nd ed., J. Brandrup and E. H. Immergut, Ed., Wiley-Interscience, New York, N. Y., 1975, Chapter II.

2. M. Kamachi, M. Kohno, D. J. Liaw, and S. Katsuki, Polym. J., 10, 69 (1978).

3. J. A. Harris, O. Hinojosa, and J. C. Arthur, Jr., J. Polym. Sci., Polym. Chem. Ed., 11, 3215 (1973).

4. C. H. Bamford, A. Bibby, and G. C. Eastmond, Polymer, 9, 629 (1968).

5. M. Iwatsuki and Y. Sakai, J. Polym. Sci., A-1, 7, 1537 (1969).

6. H. Fischer, J. Polym. Sci., B, 2, 529 (1964).

7. P. Smith, R. D. Stevens, and L. B. Gilman, J. Phys. Chem., 79, 2688 (1975). 
8. P. Smith, L. B. Gilman, and R. A. Delorenzo, J. Mag. Resonance, 10, 179 (1973).

9. S. E. Bresler, E. N. Kozbekov, V. N. Fomichev, and V. N. Shadrin, Makromol. Chem., 157, 167 (1972).

10. S. E. Bresler, E. N. Kozbekov, V. N. Fomichev, and
V. N. Shadrin, Makromol. Chem., 175, 2875 (1974).

11. M. Kamachi, D. J. Liaw, and S. Nozakura, Polym. J., 13, 69 (1981).

12. M. Kamachi, Y. Kuwae, and S. Nozakura, Polym. J., 13, 991 (1981). 